\title{
LECTOR: Towards Reengaging Students in the Educational Process Inside Smart Classrooms
}

\author{
Maria Korozi ${ }^{1(\sqrt{(})}$, Asterios Leonidis ${ }^{1}$, Margherita Antona ${ }^{1}$, \\ and Constantine Stephanidis ${ }^{1,2}$ \\ ${ }^{1}$ Foundation for Research and Technology - Hellas (FORTH), \\ Institute of Computer Science (ICS), Heraklion, Greece \\ \{korozi, leonidis, antona, cs\}@ics.forth.gr \\ ${ }^{2}$ Department of Computer Science, University of Crete, Heraklion, Greece
}

\begin{abstract}
This paper presents LECTOR, a system that helps educators in understanding when students have stopped paying attention to the educational process and assists them in reengaging the students to the current learning activity. LECTOR aims to take advantage of the ambient facilities that "smart classrooms" have to offer by (i) enabling educators to employ their preferred attention monitoring strategies (including any well-established activity recognition techniques) in order to identify inattentive behaviors and (ii) recommending interventions for motivating distracted students when deemed necessary. Furthermore, LECTOR offers an educator friendly design studio that enables teachers to create or modify the rules that trigger "inattention alarms", as well as tailor the intervention mechanism to the needs of their course by modifying the respective rules. This paper presents the rationale behind the design of LECTOR and outlines its key features and facilities.
\end{abstract}

Keywords: Smart classroom $\cdot$ Attention monitoring $\cdot$ Ambient intelligence

\section{Introduction}

In the recent past there has been growing interest in how Information and Communication technologies (ICTs) can improve the efficiency and effectiveness of education; it is acknowledged that when used appropriately, they are potentially powerful tools for advancing or even reshaping the educational process. In more details, ICTs are claimed to help expand access to information and raise educational quality by, among others, helping make learning and teaching a more engaging, active process connected to real life [27]. Learning with the use of ICTs has been strongly related to concepts such as distance learning [4], educational games [7], intelligent tutoring systems and e-learning applications [5]. Additionally, the notion of "smart classrooms", where activities are enhanced and augmented through the use of pervasive and mobile computing, sensor networks, artificial intelligence, etc. [6], has become prevalent in the past decade [30].

However, despite the fact that the educational process is continuously enriched with engaging activities, it is almost inevitable that students will get distracted either by internal stimuli (e.g., thoughts and attempts to retrieve information from memory) or 
external stimuli from the physical (e.g., visuals, sounds) or digital environment (e.g., irrelevant applications); hence, they might not always be "present" to take advantage of all the benefits that a "smart classroom" has to offer. This observation highlights the need for a mechanism that monitors the learners and when necessary, intervenes to appropriately reset attention levels.

The proposed system, named LECTOR, aims to take advantage of the ambient facilities that "smart classrooms" have to offer and enable educators to employ their preferred attention monitoring strategies (including any well-established activity recognition techniques) in order to identify inattentive behaviors and assist the educator in reengaging the students to the current learning activity. In more details, the main contributions of this work are listed below:

- Extensible mechanism for deploying various attention monitoring strategies aiming to identify inattentive behaviors

- Extensible intervention mechanism for intervening when the students are distracted from the educational process

- Educator-friendly tools for: (i) refining the inattention models and labeling newly discovered student activities, as well as (ii) creating new or modifying existing intervention strategies.

\section{Background Theory}

Attention is very often considered as a fundamental prerequisite of learning, both within and outside the classroom environment, since it plays a critical role in issues of motivation and engagement [20]. However, as passive listeners, people generally find it difficult to maintain a constant level of attention over extended periods of time, while pedagogical research reveals that attention lapses are inevitable during a lecture. McKeachie [16], suggests that student attention will drift during a passive lecture, unless interactive strategies are used. According to [31], student concentration decays in the same way during a passive lecture as does that of a human operator monitoring automated equipment, with serious implications for learning and performance. Obtaining and maintaining the students' attention is an important task in classroom management, and educators apply various techniques for this purpose, however currently no technological support is available to assist educator in monitoring students' behavior in the classroom and maximizing students' engagement at the task at hand. According to Packard [19], "classroom attention" refers to a complex and fluctuating set of stimulusresponse relationships involving curriculum materials, instructions from the teacher and some prerequisite student behaviors (e.g., looking, listening, being quiet, etc.). Such behaviors can be rigorously classified as "appropriate" and "inappropriate" [26]. Appropriate behaviors include attending to the teacher, raising hand and waiting for the teacher to respond, working in seat on a workbook, following text reading, etc., while inappropriate behaviors include (but are not limited to) getting out of seat, tapping feet, rattling papers, carrying on a conversation with other students, singing, laughing, turning head or body toward another person, showing objects or looking at another class mate. Some of the above behaviors would be in fact disruptive to some educational activities. 
However, the students should not be forced to spend their whole day not being children, but being quiet, docile, and obedient "young adults" [29]. On the contrary, learning can be more effective if students' curiosity, along with their desire to think or act for themselves, remains intact.

Attention aware systems have much to contribute to educational research and practice. These systems can influence the delivery of instructional materials, the acquisition of such materials from presentations (as a function of focused attention), the evaluation of student performance, and the assessment of learning methodologies (e.g., traditional teaching, active learning techniques, etc.) [20]. However, existing approaches [3, 17, $22,23,28]$ concentrate mainly on computer-driven educational activities. This work broadens the perspective by employing attention monitoring in a real classroom and incorporating a mechanism for suggesting improvements for the learning process; most importantly though, it empowers educators to customize or even create from scratch new inattention detection rules (e.g., "if the students whisper while the educator is writing to the whiteboard...") and intervention strategies.

\section{The Smart Classroom Behind LECTOR}

LECTOR is employed inside a technologically augmented classroom where educational activities are enhanced with the use of pervasive and mobile computing, sensor networks, artificial intelligence, multimedia computing, middleware and agent-based software $[1,13,24]$. In more details, the hardware infrastructure includes both commercial and custom-made artifacts, which are embedded in traditional classroom equipment and furniture. For example, the classroom contains a commercial touch sensitive interactive whiteboard, technologically augmented student desks [21] that integrates various sensors (e.g., eye-tracker, camera, microphone, etc.), a personal workstation and a smart watch for the teacher, as well as various ambient facilities appropriate for monitoring the overall environment and the learners' actions (e.g., microphones, user-tracking devices, etc.).

The software architecture (Fig. 1b) of the smart Classroom follows a stack-based model where the first layer, namely the AmI-Solertis middleware infrastructure [15], is responsible for (i) the collection, analysis and storage of the metadata regarding the environment's artifacts, (ii) their deployment, execution and monitoring in the AmISolertis-enabled systems to formulate a ubiquitous ecosystem. The next three layers, namely the ClassMATE, CognitOS and LECTOR frameworks, expose the core libraries and finally the remaining layer contains the educational applications. Specifically, ClassMATE [14] is an integrated architecture for pervasive computing environments that monitors the ambient environment and makes context-aware decisions; it features a sophisticated, unobtrusive, profiling mechanism in order to provide user related data to the classroom's services and applications. Furthermore, CognitOS [18] delivers a sophisticated environment for educational applications hosting able to present interventions that will be dictated by LECTOR. 


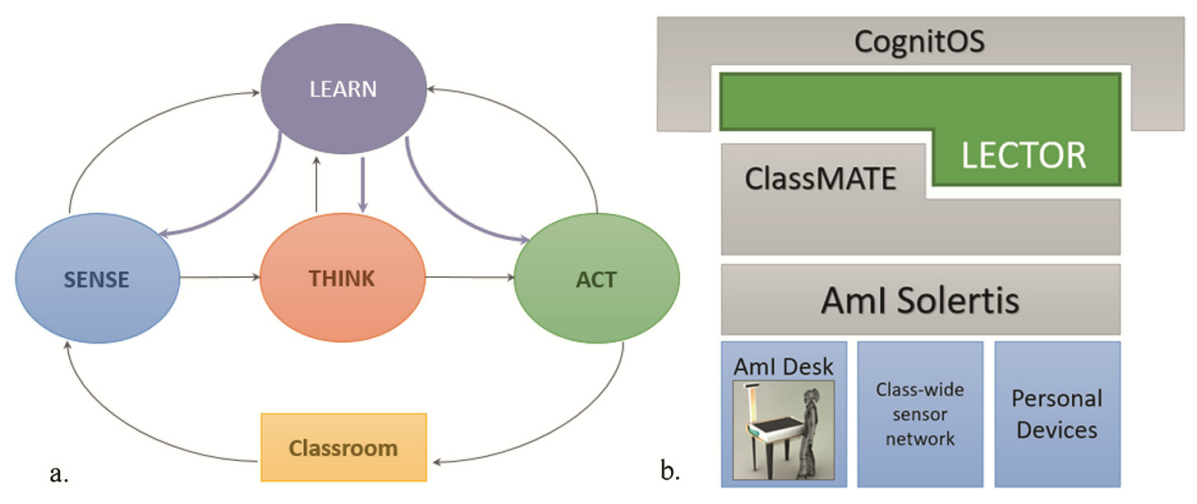

Fig. 1. (a) LECTOR's SENSE-THINK-ACT - LEARN model. (b) The software architecture of the smart classroom

\section{LECTOR Approach}

LECTOR introduces a non-invasive multimodal solution, which exploits the potential of ambient intelligence technologies to observe student actions (SENSE), provides a framework to employ activity recognition techniques for identifying whether these actions signify inattentive behavior (THINK) and intervenes -when necessary- by suggesting appropriate methods for recapturing attention (ACT). According to cognitive psychology, the sense-think-act cycle stems from the processing nature of human beings that receive input from the environment (perception), process that information (thinking), and act upon the decision reached (behavior). Such pattern became the base for many design principles regarding autonomous agents and traditional AI.

For that to be optimally achieved, the proposed system is able to make informed decisions using volatile information and reliable knowledge regarding the syllabus covered so far, the nature of the current activity, the "expected" behavior of the involved individuals towards it, the behavior of the peers, etc. The aforementioned pieces of information can be classified under the broader term of Context of Use, defined as follows: "Any information that can be used to characterize the situation of entities (i.e., whether a person, place, or object) that are considered relevant to the interaction between a user and an application, including the user and the application themselves. Context is typically the location, identity, and state of people, groups, and computational and physical objects" [8]. Based on the above, the SENSE-THINK-ACT model of LECTOR relies on an extensible modeling component to collect and expose such classroomspecific information.

This work extends the SENSE-THINK-ACT model by introducing the notion of LEARN (Fig. 1a). The fact that the nature of this system enables continuous observation of student activities creates the foundation for a mechanism that provides updated knowledge to the decision-making components. In more details, the LEARN-ing mechanism is able to (i) assess decisions that resulted in negative outcomes in the past (e.g., inattention levels remain high or deteriorate after introducing a mini-quiz intervention 
during a math course) and (ii) incorporate knowledge provided by the teacher (e.g., disambiguation of student behavior, rejection of suggested intervention during a specific course, etc.).

\subsection{Motivating Scenarios}

Monitoring the Attention Levels of an Entire Classroom. On Monday morning the history teacher, Mr. James, enters the classroom and announces that the topic of the day will be the "Battle of Gaugamela". During the first 15 min the students pay attention to the teacher who narrates the story; soon enough, the students start losing interest and demonstrate signs of inattentive behavior. In more details, John is browsing through the pages of a different book, Mary and Helen are whispering to each other, Peter stares out the window and Mike struggles to keep his eyes open. When identifying that the entire classroom demonstrates signs of inattention, the system recommends that the lecture should be paused and that a mini quiz game should be started. The teacher finishes up his sentence and decides to accept this intervention. After his confirmation, a set of questions relevant to the current topic is displayed on the classroom board, while their difficulty depends on both the students' prior knowledge and the studied material so far. During use, the system identifies the topics with the lowest scores and notifies the teacher to explain them more thoroughly. As soon as the intervention ends, Mr. James resumes the lecture. At this point, the students' attention is reset and they begin to pay attention to the historical facts. As a result, the quiz not only restored their interest, but also resulted in deeper learning.

Monitoring the Attention Levels of an Individual Student. During the geography class Kate is distracted by a couple of students standing outside the window. The system recognizes that behavior and takes immediate action to attract her interest back on the lecture. To do so, it displays pictures relevant to the current topic on her personal workstation while a discreet nudge attracts her attention. A picture displaying a dolphin with weird colors swimming in the waters of Amazon makes her wondering how it is possible for a dolphin to survive in a river; she patiently waits for the teacher to complete his narration to ask questions about that strange creature. That way, Kate becomes motivated and starts paying attention to the presentation of America's rivers. At the same time, Nick is drawing random pictures on his notebook and seems to not pay attention to the lecture; however, the system already knows that he concentrates more easily when doodling, and decides not to interpret that behavior as inattention.

\subsection{Context of Use}

LECTOR's decision-mechanisms are heavily dependent on contextual information to (i) identify the actual conditions (student status, lecture progress, task at hand, etc.) that prevail in a smart classroom at any given time and (ii) act accordingly. The term context has been used broadly with a variety of meanings for context-aware applications in pervasive computing [9]. The authors in [10] refer to contexts as any information that can be detected through low-level sensor readings; for instance, in a home environment 
those reading include the room that the inhabitant is in, the objects that the inhabitant interacts with, whether the inhabitant is currently mobile, the time of the day when an activity is being performed, etc.

However, in a smart classroom contextual awareness goes beyond data collected from sensors. Despite the fact that sensorial readings are important for recognizing student activities, they are inadequate to signify inattention without information regarding the nature of the current course, the task at hand, the characteristics of the learner, etc. This work employs the term Physical Context (PC) to indicate data collected from sensors, while the term Virtual Learning Context (VLC) is used for any static or dynamic information regarding the learning process (e.g., student profile, course related information, etc.) [32].

The exploitation of such contextual information can improve the performance of the THINK component, which employs activity recognition strategies in order to identify student activities and classify them as inattentive or not. Despite the fact that activity recognition mainly relies on sensor readings to detect student activities, the Virtual Learning Context (VLC) is critical to interpret inattention indicators correctly; as an example, in general excess noise indicates that students talk to each other instead of listening to the teacher; however, this is irrelevant during the music class.

Furthermore, VLC is essential for the ACT component; when the system decides to intervene in order to reset students' attention, the selection of the appropriate intervention type depends heavily on the context of use (syllabus covered so far, remaining time, etc.). As an example, if an intervention occurs during the first ten minutes of a lecture, where the main topic has not been thoroughly analyzed by the teacher yet, the system starts a short preview that briefly introduces the lecture's main points using entertaining communication channels (e.g., multimedia content).

\subsection{Sensorial Data}

LECTOR is deployed in a "smart classroom" that incorporates infrastructure able to monitor the learners' actions and provide the necessary input to the decision-making components for estimating their attention levels. To ensure scalability, this work is not bound to certain technological solutions; it embraces the fundamental concept of Ambient Intelligence that expects environments to be dynamically formed as devices constantly change their availability. As a consequence, a key requirement is to ensure that new sensors and applications can be seamlessly integrated (i.e., extensibility). In order to do so, LECTOR relies on the AmI-Solertis framework, which provides the necessary functionality for the intercommunication and interoperability of heterogeneous services hosted in the smart classroom.

As regards the supported input sources, they range from simple converters (or even chains of converters) that measure physical quantities and convert them to signals, which can be read by electronic instruments, to software components (e.g., a single module, an application, a suite of applications, etc.) that monitor human computer interaction and data exchange. However, a closer look at the sensorial data reveals that it is not the actual value that matters, but rather the meaning of that value. For instance, the attention 
recognition mechanism does not need to know that a student has turned his head $23^{\circ}$ towards south but that he stares out of the window.

Subsequently, LECTOR equips the developers with an authoring tool that enables them to provide the algorithms that translate the raw data into meaningful high-level objects. In more details, through an intuitive wizard (Fig. 2) the developers (i) define the contextual properties (e.g., Speech, Feelings, Posture, etc.) that will be monitored by the system, (ii) specify the attributes of those properties (e.g., level, rate, duration, etc.) and (iii) develop the code that translates the actual values coming directly from the sensors/applications to those attributes. The in-vitro environment where LECTOR is deployed employs the following ambient facilities:

- Eye-trackers to observe students' fixations during studying on a personal computer (e.g., reading a passage, solving an exercise) to determine the attention level (e.g., stares at an insignificant area of the screen), the weaknesses (e.g., the student keeps reading the same sentence over and over again), the interests (e.g., fascinated with wild life) and the learning styles (e.g., attempts the easier assignments first) of each student. The same information can be also provided by custom educational software (i.e., CognitOS).

- Sophisticated cameras (e.g., RGB-D camera such as Microsoft Kinect) that track the head pose of the learner and are used as a surrogate for gaze. The combination of eye-tracking and head pose tracking algorithms offers an accurate overview of what the students are looking at on the computer screen and on whom or what they are focused on (e.g., teacher, class board, etc.). Moreover, the use of cameras is ideal for tracking the body posture and the direction of an individual student, especially when taking into consideration that they constantly move even while seated. Besides learners' orientation, camera input also enables the identification of specific gestures that indicate whether a student is paying attention to the lecture or not (e.g., a student raising his hand). Finally, they can be used to analyze whether the students' capabilities are compromised due to feelings of fatigue (i.e., Drowsiness, Falling Asleep).

- Microphones are placed on the teacher's and students' desks to identify who is speaking at any time and the overall noise levels of the classroom, which can reliably indicate inattentive behavior on behalf of the students.

- Pressure-sensitive sensors on each learner's chair to identify whether the student is seated or not. This information when combined with data received from strategically placed distance and motion sensors (e.g., near the class board, near the teacher's desk), introduces a primitive localization technique that can be used to estimate the location and the purpose of a "missing" individual (e.g., a student is off the desk but near the board thus solving an exercise).

- Wearable sensors that can be used to monitor the students' physiological signals (e.g., heart rate, EDA, etc.).

LECTOR currently uses the aforementioned ambient facilities to monitor some physical characteristics of the students and teachers and translates them, in a contextdependent manner, into specific activities classified under the following categories: Focus, Speech, Location, Posture and Feelings, which are considered appropriate cues that might signify inattention $[2,11,19,25]$. 

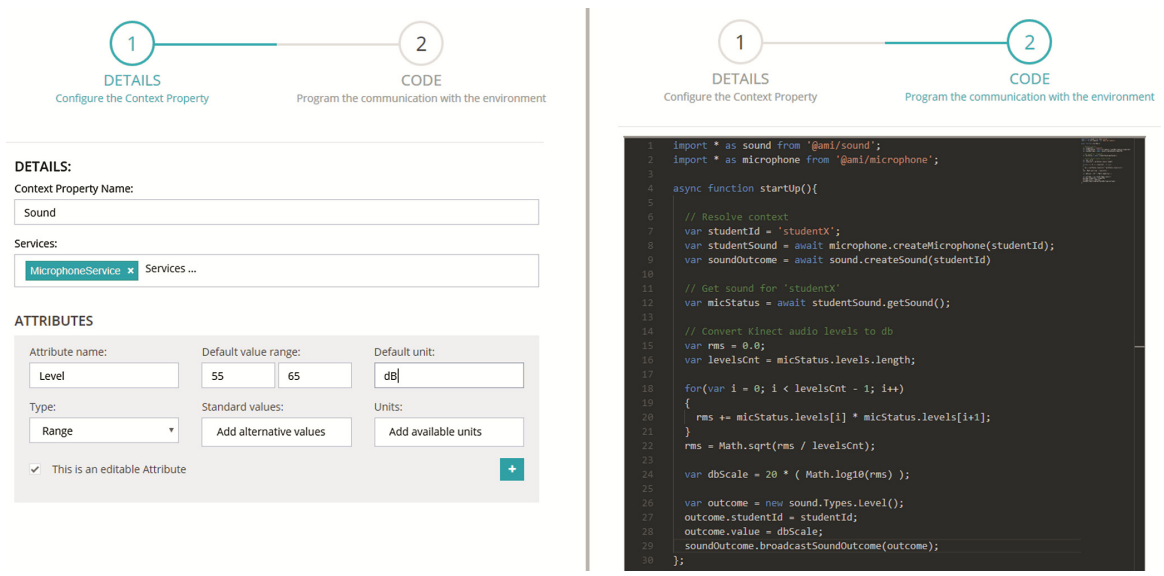

Fig. 2. Snapshot from the developers' authoring tool, displaying the process of defining the 'SOUND' contextual property.

\subsection{Inattention Alarms}

LECTOR's THINK component (Fig. 3) is responsible for identifying the students who show signs of inattention. Towards such objective, it constantly monitors their actions in order to detect (sub-) activities that imply distraction and loss of attention. The decision logic that dictates which behaviors signify inattention is expressed via high-level rules in the "Attention rule set", which combines various contextual parameters to define the conditions under which a student is considered distracted. There are two type of rules in the "Attention rule set": (i) rules that denote human activities or sub-activities (e.g., talking, walking, sitting, etc.) and provide input to (ii) rules that signify inattentive behaviors (e.g., disturb, chat, cheat, etc.). Through an educator-friendly authoring tool, namely LECTORstudio [12], the teachers have the opportunity to create or modify the latter, while -due to their complexity- they can only fine-tune the rules that denote human (sub-) activities.

Whenever a stimulus is detected by the SENSE component, the THINK component initiates an exploratory process to determine whether the incoming event indicates that the student(s) has lost interest in the learning process or not. In order to do so, it employs the appropriate attention recognition strategies based on the "Attention rule set". Finally, at the end of the exploratory process, if the result points to inattentive behavior, SENSE appropriately informs the ACT component which undertakes to restore student engagement by selecting an appropriate intervention.

Figure 4 presents the graphical representation of a rule describing the activity "SHOUTING", as created in LECTORstudio. Specifically, the purpose of this rule is to create an exception for the Music course, where students sing, thus raising the noise levels of the classroom higher than usual; in that case, the activity "SHOUTING" should be identified when the sound volume captured through the class microphone exceeds the value of $82 \mathrm{~dB}$. 


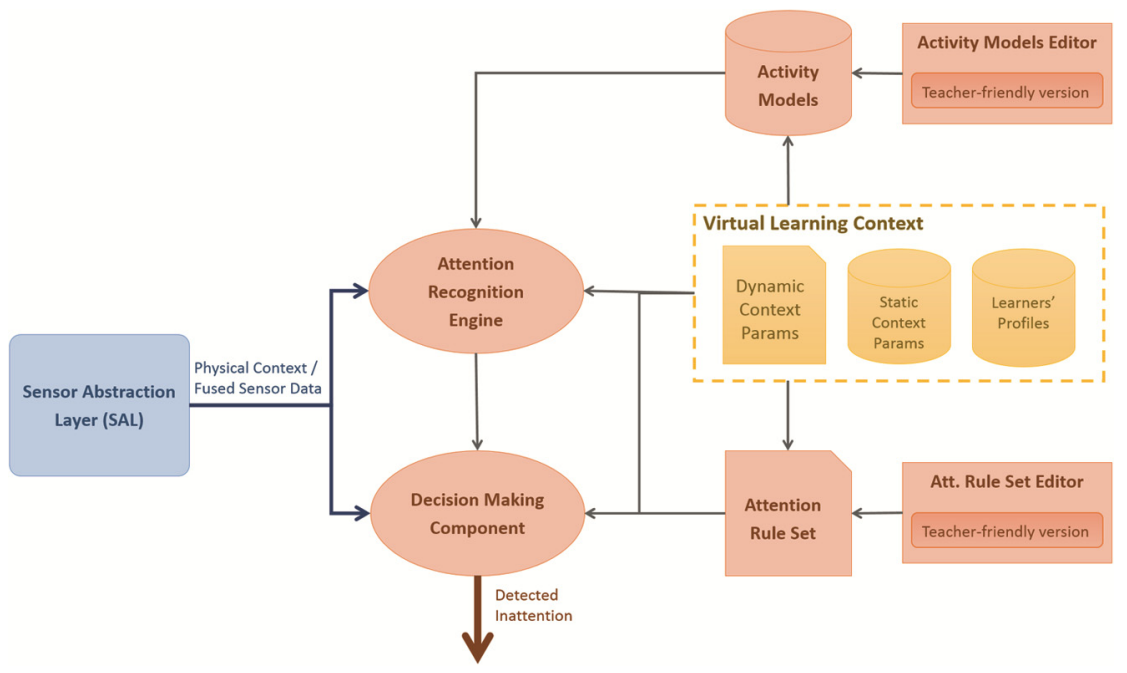

Fig. 3. LECTOR's THINK component.
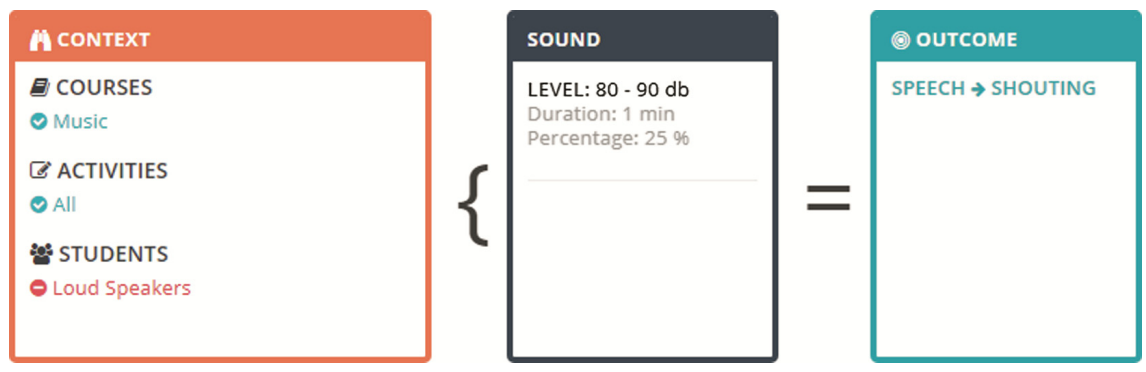

Fig. 4. A rule describing the activity "SHOUTING", as created in LECTORstudio.

\subsection{Intervention Rules}

As soon as inattentive behavior is detected, the ACT component (Fig. 5) initiates an exploratory process to identify the most appropriate course of action. Evidently, selecting a suitable intervention and its proper presentation (appropriate for the device where it will be delivered) is not a straightforward process, as it requires in-depth analysis of both the learners' profile and the contextual information regarding the current course. The first step is to consult the "Intervention rule set", which, similarly to the "Attention rule set", is comprised of high-level rules describing the conditions under which each intervention should be selected (e.g., if all students are distracted during the math course, recommend an interactive task like a mini-quiz) as well as the appropriate means of presentation (e.g., if a mini-quiz is selected and the intervention is intended for all students, display it to the classroom interactive board). 


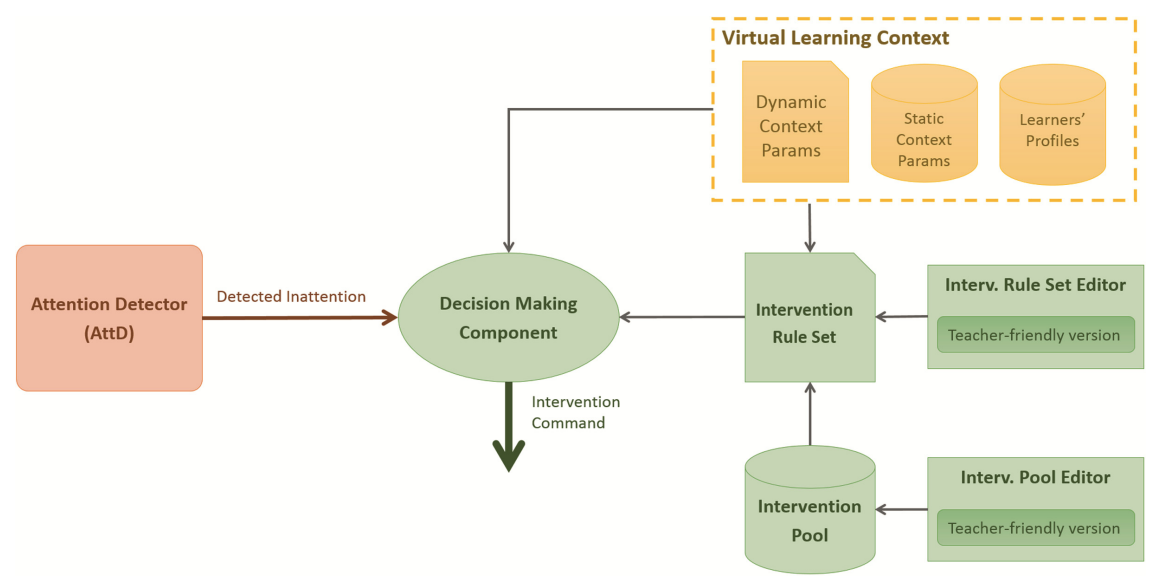

Fig. 5. LECTOR's ACT component.

Each intervention rule, upon evaluation, points to a certain intervention strategy into the "Interventions' Pool" (IP). The IP includes high-level descriptions of the available strategies, along with their low-level implementation descriptions. Furthermore, since inattention can originate either from a single student or the entire classroom, the ACT component should be able to evaluate and select strategies targeting either an individual student or a group of students (even the entire class). To this end, the "Interventions' Pool" should contain interventions of both types, and the decision logic should be able to select the most appropriate one. After selecting the appropriate intervention, the system personalizes its content to the targeted student and converts it to a form suitable for the intended presentation device.

LECTORstudio also permits the teachers to tailor the intervention mechanism to the needs of their course by modifying the "Intervention Rule Set". In more details, a teacher can create custom interventions, customize existing ones in terms of their content, change the conditions under which an intervention is initiated (e.g., the percentage of distracted students), etc.

\subsection{Intervention Assessment}

Both the THINK and ACT components are able to "learn" from previous poor decisions and refine their logic, while they are open to expert suggestions that can override their defaults. In order to introduce the notion of LEARN, LECTOR provides mechanisms that modify the decision-making processes by correlating knowledge gathered through attention monitoring with student performance and expert input.

To this end, the LEARN component is able to assess the regression of students' attention lapses -through the respective student profile component- with a formerly applied intervention to identify whether it had positive results or it failed to reset attention. In more details, if the system estimates that a particular intervention will reset attention in the context of a specific course and applies it, then after a reasonable amount of time it re-calculates the current attention levels; if it still detects that the students are 
not committed to the learning process, then the selected recommendation is marked as ineffective in that context. Hence, the ACT component is informed so as to modify its decision logic accordingly, and from that point forward select different interventions for that particular course instead of the one that was proven to be unsuccessful.

On top of the automatic application of active learning interventions, the system also permits additions, modifications, cancellations and ranking of the selected interventions. This allows the teacher to have the final say regarding the lecture format. To this end, the LEARN component takes into consideration the teacher's input and appropriately inform the ACT component so as to refine the intervention rule set and offer more effective alternatives when necessary. In more details, the teacher should be able to: (i) change the recommended intervention with a more appropriate one (e.g., quiz, multimedia presentation, discussion, etc.), (ii) rank the recommendation and (iii) abort the intervention in case it disrupts the flow of the course.

\section{Conclusions and Future Work}

LECTOR provides a framework and an educator-friendly design studio for the smart classroom in order to improve the educational process. For that to be achieved, it equips the environment with a system that is able to monitor the learners' attention levels depending on rules created by the teachers themselves and intervenes, when necessary, to (i) provide a motivating activity to a distracted student or (ii) suggest an alternative pedagogy that would be beneficial for the entire classroom (e.g., by motivating individuals or suggesting different lecture formats, etc.).

Future work includes full-scale evaluation experiments in order validate the system's efficacy and usability. In particular, two types of user-based experiments will be conducted: (i) Experiments for assessing the usability of the design studio for the teacher's. (ii) Experiments for evaluating the system as a whole. These experiments will be conducted for an extended period of time inside the smart classroom environment, where students and teachers will be engaged with several educational activities while the system will monitor the learners' attention levels throughout the entire process and intervene when necessary. The results of this evaluation will be used to identify whether the system can: (a) appropriately adapt its behavior in order to respect teachers' input, and (b) positively affect -through the delivery of personalized interventions- the students' motivation level and overall performance.

\section{References}

1. Antona, M., Leonidis, A., Margetis, G., Korozi, M., Ntoa, S., Stephanidis, C.: A student-centric intelligent classroom. In: Keyson, D.V., Maher, M.L., Streitz, N., Cheok, A., Augusto, J.C., Wichert, R., Englebienne, G., Aghajan, H., Kröse, B.J.A. (eds.) AmI 2011. LNCS, vol. 7040, pp. 248-252. Springer, Heidelberg (2011). https://doi.org/10.1007/978-3-642-25167-2_33

2. Ba, S.O., Odobez, J.-M.: Recognizing visual focus of attention from head pose in natural meetings. IEEE Trans. Syst. Man Cybern. Part B Cybern. 39(1), 16-33 (2009)

3. Barrios, V.M.G., et al.: AdELE: a framework for adaptive e-learning through eye tracking. In: Proceedings of IKNOW, pp. 609-616 (2004) 
4. Bates, A.T.: Technology, e-Learning and Distance Education. Routledge, London (2005)

5. Brooks, C., Greer, J., Melis, E., Ullrich, C.: Combining its and e-learning technologies: opportunities and challenges. In: Ikeda, M., Ashley, K.D., Chan, T.-W. (eds.) ITS 2006. LNCS, vol. 4053, pp. 278-287. Springer, Heidelberg (2006). https://doi.org/10.1007/11774303_28

6. Cook, D.J., Das, S.K.: How smart are our environments? An updated look at the state of the art. Pervasive Mob. Comput. 3(2), 53-73 (2007)

7. Cross, N., Roy, R.: Engineering Design Methods. Wiley, New York (1989)

8. Dey, A.K., et al.: A conceptual framework and a toolkit for supporting the rapid prototyping of context-aware applications. Hum. Comput. Interact. 16(2), 97-166 (2001)

9. Henricksen, K., Indulska, J.: Developing context-aware pervasive computing applications: models and approach. Pervasive Mob. Comput. 2(1), 37-64 (2006)

10. Hong, X., et al.: Evidential fusion of sensor data for activity recognition in smart homes. Pervasive Mob. Comput. 5(3), 236-252 (2009)

11. Hwang, K.-A., Yang, C.-H.: Automated inattention and fatigue detection system in distance education for elementary school students. J. Educ. Technol. Soc. 12(2), 22-35 (2009)

12. Korozi, M., et al.: LECTORstudio: creating inattention alarms and interventions to reengage the students in the educational process. In: Proceedings of the 10th Annual International Conference of Education, Research and Innovation (2017)

13. Leonidis, A., et al.: A glimpse into the ambient classroom. Bull. IEEE Tech. Comm. Learn. Technol. 14(4), 3-6 (2012)

14. Leonidis, A., et al.: ClassMATE: enabling ambient intelligence in the classroom. World Acad. Sci. Eng. Technol. 66, 594-598 (2010)

15. Leonidis, A., et al.: The AmI-Solertis system: creating user experiences in smart environments. In: Proceedings of the 13th IEEE International Conference on Wireless and Mobile Computing, Networking and Communications (2017)

16. McKeachie, W., Svinicki, M.: McKeachie's Teaching Tips. Cengage Learning, Boston (2013)

17. Merten, C., Conati, C.: Eye-tracking to model and adapt to user meta-cognition in intelligent learning environments. In: Proceedings of the 11th International Conference on Intelligent User Interfaces, pp. 39-46. ACM (2006)

18. Ntagianta, A., et al.: CognitOS: a student-centric working environment for an attention-aware intelligent classroom. In: Proceedings of the 20th International Conference on HumanComputer Interaction. Springer, Heidelberg (2017, submitted)

19. Packard, R.G.: The control of "classroom attention": a group contingency for complex behavior. J. Appl. Behav. Anal. 3(1), 13-28 (1970)

20. Rapp, D.N.: The value of attention aware systems in educational settings. Comput. Hum. Behav. 22(4), 603-614 (2006)

21. Savvaki, C., Leonidis, A., Paparoulis, G., Antona, M., Stephanidis, C.: designing a technologyaugmented school desk for the future classroom. In: Stephanidis, C. (ed.) HCI 2013. CCIS, vol. 374, pp. 681-685. Springer, Heidelberg (2013). https://doi.org/10.1007/978-3-642-39476-8_137

22. Sibert, J.L., et al.: The reading assistant: eye gaze triggered auditory prompting for reading remediation. In: Proceedings of the 13th Annual ACM Symposium on User Interface Software and Technology, pp. 101-107. ACM (2000)

23. Slykhuis, D.A., et al.: Eye-tracking students' attention to PowerPoint photographs in a science education setting. J. Sci. Educ. Technol. 14(5), 509-520 (2005)

24. Stephanidis, C., et al.: Pervasive computing @ ICS-FORTH. In: Workshop Pervasive Computing @ Home (2008)

25. Sylwester, R.: How emotions affect learning. Educ. Leadersh. 52(2), 60-65 (1994) 
26. Thomas, D.R., et al.: Production and elimination of disruptive classroom behavior by systematically varying teacher's behavior. J. Appl. Behav. Anal. 1(1), 35-45 (1968)

27. Tinio, V.L.: ICT in Education. e-ASEAN Task Force (2003)

28. Wang, H., et al.: Empathic tutoring software agents using real-time eye tracking. In: Proceedings of the 2006 Symposium on Eye Tracking Research \& Applications, pp. 73-78. ACM (2006)

29. Winett, R.A., Winkler, R.C.: Current behavior modification in the classroom: be still, be quiet, be docile. J. Appl. Behav. Anal. 5(4), 499-504 (1972)

30. Xu, P., Han, G., Li, W., Wu, Z., Zhou, M.: Towards intelligent interaction in classroom. In: Stephanidis, C. (ed.) UAHCI 2009. LNCS, vol. 5616, pp. 150-156. Springer, Heidelberg (2009). https://doi.org/10.1007/978-3-642-02713-0_16

31. Young, M.S., et al.: Students pay attention! Combating the vigilance decrement to improve learning during lectures. Act. Learn. High Educ. 10(1), 41-55 (2009)

32. Zhang, D., et al.: Survey on context-awareness in ubiquitous media. Multimedia Tools Appl. 67(1), 179-211 (2013)

Open Access This chapter is licensed under the terms of the Creative Commons Attribution 4.0 International License (http://creativecommons.org/licenses/by/4.0/), which permits use, sharing, adaptation, distribution and reproduction in any medium or format, as long as you give appropriate credit to the original author(s) and the source, provide a link to the Creative Commons license and indicate if changes were made.

The images or other third party material in this chapter are included in the chapter's Creative Commons license, unless indicated otherwise in a credit line to the material. If material is not included in the chapter's Creative Commons license and your intended use is not permitted by statutory regulation or exceeds the permitted use, you will need to obtain permission directly from the copyright holder. 